\title{
Prevalence of airflow limitation in outpatients with cardiovascular diseases in Japan
}

\author{
This article was published in the following Dove Press journal: \\ International Journal of COPD \\ 29 May 2014 \\ Number of times this article has been viewed
}

\section{Katsuya Onishi' \\ Daisuke Yoshimoto ${ }^{2}$ \\ Gerry W Hagan ${ }^{3}$ \\ Paul W Jones ${ }^{4}$}

'Onishi Heart Clinic, Mie, ${ }^{2}$ Medical Affairs Respiratory, GlaxoSmithKline KK, Tokyo, Japan; ${ }^{3}$ Independent Consultant, Marbella, Spain; ${ }^{4}$ Division of Clinical Science, St George's, University of London, London, UK
Correspondence: Katsuya Onishi

Onishi Heart Clinic, 343I-5 Handa,

Tsu, Mie 5I4-0823, Japan

Tel +8I 592252597

Fax +8I 592253033

Email katsu08I2@way.ocn.ne.jp
Background and objectives: Cardiovascular disease (CVD) and chronic obstructive pulmonary disease (COPD) commonly coexist and share common risk factors. The prevalence of COPD in outpatients with a smoking history and CVD in Japan is unknown. The aim of this study was to determine the proportion of Japanese patients with a smoking history being treated for CVD who have concurrent airflow limitation compatible with COPD. A secondary objective was to test whether the usage of lung function tests performed in the clinic influenced the diagnosis rate of COPD in the patients identified with airflow limitation.

Methods: In a multicenter observational prospective study conducted at 17 centers across Japan, the prevalence of airflow limitation compatible with COPD (defined as forced expiratory volume $(\mathrm{FEV})_{1} / \mathrm{FEV}_{6}<0.73$, by handheld spirometry) was investigated in cardiac outpatients $\geq 40$ years old with a smoking history who routinely visited the clinic for their CVD. Each patient completed the COPD Assessment Test prior to spirometry testing.

Results: Data were available for 995 patients with a mean age of $66.6 \pm 10.0$ years, of whom 95.5\% were male. The prevalence of airflow limitation compatible with COPD was $27.0 \%$ $(n=269)$, and $87.7 \%$ of those patients $(n=236)$ did not have a prior diagnosis of COPD. The prevalence of previously diagnosed airflow limitation was higher in sites with higher usage of lung function testing $(14.0 \%, 15.2 \%$ respectively) compared against sites where it is performed seldom (11.1\%), but was still low.

Conclusion: The prevalence of airflow limitation in this study indicates that a quarter of outpatients with CVD have COPD, almost all of whom are undiagnosed. This suggests that it is important to look routinely for COPD in CVD outpatients.

Keywords: COPD, CVD, handheld spirometer, lung function, diagnosis

\section{Introduction}

The prevalence of chronic obstructive pulmonary disease (COPD) is expected to increase globally. ${ }^{1,2}$ There is significant underdiagnosis of COPD in Japan, partly because spirometry testing is limited $^{3}$ and symptoms appear progressively, becoming obvious only when lung function is already significantly impaired. ${ }^{4}$ Previous data have shown that spirometry screening during medical checkups can identify many patients with COPD who are not aware of this disease. ${ }^{5}$

COPD is associated with increased risk of cardiovascular disease (CVD) and, in subjects with CVD, the coexistence of COPD is associated with raised morbidity, including a worsening of symptoms and more frequent hospitalizations. ${ }^{6,7}$ In addition, cardiovascular deaths are a significant cause of mortality in COPD patients. ${ }^{9}$ 
Surveys have been performed to look at the prevalence of COPD in patients consulting medical institutions specializing in circulatory diseases. In Spain, airflow limitation was detected in $19 \%$ of patients with CVD. ${ }^{10}$ In Japan, from patients consulting the cardiovascular department of a university hospital, the prevalence of airflow limitation ranged from $10 \%-25 \%$, depending on the primary conditions of the sampled patients. ${ }^{11,12}$ However, the possibility of a significant risk of the comorbidity of COPD and CVD is not widely accepted in Japan.

The combination of COPD with CVD is known to be associated with poorer prognosis, ${ }^{7-9}$ largely attributed to increased systemic inflammation. ${ }^{13}$ Observational data have suggested that slowing COPD progression may help to reduce cardiovascular morbidity and mortality, which are themselves associated with more severe pulmonary symptoms. ${ }^{14}$ Exacerbations of COPD have been demonstrated to increase the risk of myocardial infarction and stroke, ${ }^{15}$ and COPD patients with CVD have significantly higher risk of COPD exacerbations and increased costs than COPD patients without CVD. ${ }^{16}$ Therefore, there is a good rationale for case-finding for COPD, in order to manage the disease as early as possible, prevent exacerbations, and reduce the risk of cardiovascular events. ${ }^{17}$

The aim of this study was to determine the proportion of Japanese patients with a smoking history being treated for cardiovascular disease who have concurrent airflow limitation compatible with COPD. A secondary objective was to test whether the usage of respiratory function tests performed in the clinic influenced the diagnosis rate of COPD in patients identified with airflow limitation.

\section{Methods}

\section{Study design}

This study was a multicenter prospective observational study conducted at 17 clinics in Japan; in consecutive outpatients with a smoking history who routinely visited the clinic for their CVD. All patients gave written consent to participate in the study. Patients who were $\geq 40$ years old and had a smoking history of $\geq 10$ pack-years were enrolled in the study. Patients with hypertension were required to have received drug therapy for at least 1 year to be included. Any patient who had difficulty in performing spirometry on the day of the study visit, due to an acute respiratory infection, was required to return when the infection had resolved without the use of bronchodilator. Patients diagnosed with bronchial asthma were excluded.

Assessments were made at a single visit. Patients were asked to complete the COPD Assessment Test (CAT) questionnaire $^{18}$ and a pulmonary function test, using a handheld spirometer: Hi-Checker (Takara Tsusho Co, Ltd, Tokyo, Japan) or Vitalograph (Vitalograph Inc., Lenexa, $\mathrm{KS}$, USA). Forced expiratory volume in 1 second $\left(\mathrm{FEV}_{1}\right)$, and in 6 seconds $\left(\mathrm{FEV}_{6}\right)$ was measured prior to bronchodilator use. A predicted $\mathrm{FEV}_{1}$ value was calculated according to the formula used by the Lung Physiology Committee of the Japanese Respiratory Society. ${ }^{19}$ Airflow limitation was defined as $\mathrm{FEV}_{1} / \mathrm{FEV}_{6}$ ratio $<0.73$. The $\mathrm{FEV}_{1} / \mathrm{FEV}_{6}$ ratio can be used as a valid alternative for $\mathrm{FEV}_{1}$ /forced vital capacity (FVC) in the diagnosis of airway obstruction. ${ }^{20}$ Demography and details of concurrent diseases and medications were collected. Chronic bronchitis was defined as the presence of sputum for at least 3 months of the year, for more than 2 consecutive years.

\section{Analysis}

The primary analysis set was the per protocol population. Study centers were stratified into three subsets based on the usage of pulmonary function tests. This was decided at the discretion of the cardiologists who participated in the study. Data analyses were performed for each subset. The subsets were classified as centers that performed pulmonary function tests often ( $\geq 10$ times/month), less often ( $2-10$ times/month), and seldom $(<2$ times/month). A stratified analysis was performed based on demographic and other baseline characteristics. The mean values and $95 \%$ confidence intervals were calculated for $\mathrm{FEV}_{1}, \mathrm{FEV}_{6}$, and percentage of predicted $\mathrm{FEV}_{1}$ and $\mathrm{FEV}_{1} /$ $\mathrm{FEV}_{6}$ values. Differences between groups were statistically analyzed by the chi-squared test. A $P$-value $<0.05$ was considered statistically significant.

\section{Results}

A total of 1,001 subjects were enrolled in the study. The study population consisted of 995 subjects for whom spirometry data and CAT assessment were available; six patients were removed due to a protocol violation or an inappropriate spirometry procedure.

The baseline demographics are shown in Table 1. The mean age of the study population was $66.6 \pm 10.0$ years, and the majority of patients were male (95.5\%). Hypertension was the most common CVD, which was reported in $74.0 \%$ of patients - the second highest CVD being arrhythmia (20.1\%).

The prevalence of airflow limitation in this study was $27.0 \%(n=269)$. Of these, $87.7 \%(n=236)$ did not have a previous diagnosis of COPD (Table 2). At the discretion of the consulting cardiologist centers were grouped by their usage of lung function tests. Four sites were classed as "often" 
Table I Baseline demographics of the per protocol population

\begin{tabular}{|c|c|}
\hline & $\mathrm{n}=995$ \\
\hline Mean age (SD); years & $66.6(9.96)$ \\
\hline Male; n (\%) & $950(95.5)$ \\
\hline Current smoker; n (\%) & $273(27.4)$ \\
\hline Ex-smoker; n (\%) & $722(72.6)$ \\
\hline Smoking pack-years; mean (SD) & $43.1(26.08)$ \\
\hline BMI; mean (SD) & $24.64(3.48)$ \\
\hline Diagnosed COPD; n (\%) & $48(4.8)$ \\
\hline Chronic sputum; n (\%) & $203(20.4)$ \\
\hline $\mathrm{FEV}_{1}$; mean (SD) & $2.29(0.70)$ \\
\hline $\mathrm{FEV}_{6}$; mean (SD) & $2.97(0.79)$ \\
\hline CAT score; mean (SD) & $6.9(5.8)$ \\
\hline Myocardial infarction; n (\%) & I58 (I5.9) \\
\hline Hypertension; n (\%) & $736(74.0)$ \\
\hline Chronic heart failure; n (\%) & II $3(1 \mathrm{I} .4)$ \\
\hline Angina; n (\%) & $192(19.3)$ \\
\hline Arrhythmia; n (\%) & $200(20.1)$ \\
\hline Stroke; n (\%) & $48(4.8)$ \\
\hline Arteriosclerosis; n (\%) & $24(2.4)$ \\
\hline Other CVD; n (\%) & $187(18.8)$ \\
\hline I CVD; n (\%) & $576(57.9)$ \\
\hline 2 CVD; n (\%) & $253(25.4)$ \\
\hline 3 CVD; n (\%) & $108(10.9)$ \\
\hline 4 CVD or more; $n(\%)$ & $58(5.8)$ \\
\hline
\end{tabular}

Abbreviations: BMI, body mass index; CVD, cardiovascular disease; COPD, chronic obstructive pulmonary disease; SD, standard deviation; CAT, COPD assessment test; $\mathrm{FEV}_{1}$, forced expiratory volume in I second; $\mathrm{FEV}_{6}$, forced expiratory volume in 6 seconds.

( $\mathrm{n}=184$ patients), three sites as "less often" ( $\mathrm{n}=182$ patients), and ten sites as "seldom" ( $n=629$ patients). The prevalence of airflow limitation, split by the usage of lung function test, shows that even at sites categorized as "often", the level of undiagnosed COPD was high. But it was lower than at the sites classified as "seldom" (Table 2).

Table 2 The number of patients with airflow limitation and a previous diagnosis of COPD

\begin{tabular}{lll}
\hline & \multicolumn{2}{l}{$\begin{array}{l}\text { Total subjects in per protocol population } \\
(\mathbf{n = 9 9 5 )}\end{array}$} \\
\cline { 2 - 3 } & $\begin{array}{l}\text { Airflow limitation } \\
\text { (prevalence of COPD) } \\
\mathbf{n = 2 6 9}\end{array}$ & $\begin{array}{l}\text { Previous diagnosis } \\
\text { of COPD } \\
\mathbf{n = 3 3}\end{array}$ \\
\hline Total & $27.0 \%$ & $12.3 \%$ \\
Split by age range & \\
$40-49$ years & $5.1 \%$ & $0 \%$ \\
$50-59$ years & $16.6 \%$ & $3.8 \%$ \\
$60-69$ years & $21.4 \%$ & $12.5 \%$ \\
$>70$ years & $39.4 \%$ & $13.8 \%$ \\
Lung function tests performed* & $14.0 \%$ \\
Often & $23.4 \%$ & $15.2 \%$ \\
Less often & $25.3 \%$ & $11.1 \%$ \\
Seldom & $28.6 \%$ &
\end{tabular}

Note: *Split at the discretion of the consulting cardiologist. Abbreviation: COPD, chronic obstructive pulmonary disease.
The mean percentage of predicted $\mathrm{FEV}_{1}$ of the total study population was $62.5 \% \pm 17.8 \%$. Compared against the Global initiative for chronic Obstructive Lung Disease (GOLD) classification ${ }^{26}$ (the majority of patients having an FEV , between 50\%-79\%; GOLD Grade 2), 20\% of patients had lung function less than $50 \%$ of predicted $\mathrm{FEV}_{1}$. The values in this study are prebronchodilator, not postbronchodilator (as in GOLD) (Figure 1).

The demographic characteristics of patients who had airflow limitation, or did not, are shown in Table 3 . In the subjects with airflow limitation, significant differences were observed, with lower body mass index, greater age, more chronic bronchitic symptoms, and higher CAT score. There was no significant difference in the number of patients between airflow limitation and no airflow limitation, within the CVDs of ischemic heart disease, chronic heart failure, and arrhythmias (Table 3). Percentages of patients with airflow limitation in each CVD are shown in Table 4.

\section{Discussion}

Airflow limitation compatible with COPD was present in $27.0 \%$ of outpatients with CVD and a history of smoking, with almost all of them being undiagnosed prior to this study. This prevalence estimate is in agreement with previous studies, which have reported that the prevalence of COPD is in the range $12 \%-34 \%$ amongst patients with coronary artery disease. ${ }^{10,21-24}$ In a study conducted by Soriano et al, ${ }^{10}$ the prevalence of airway limitation was $19.2 \%$ in subjects with CVD. It was not a Japanese study and lacked criteria for age and smoking history; the prevalence was lower than our study result. In studies conducted by Wada et $\mathrm{al}^{11}$ and

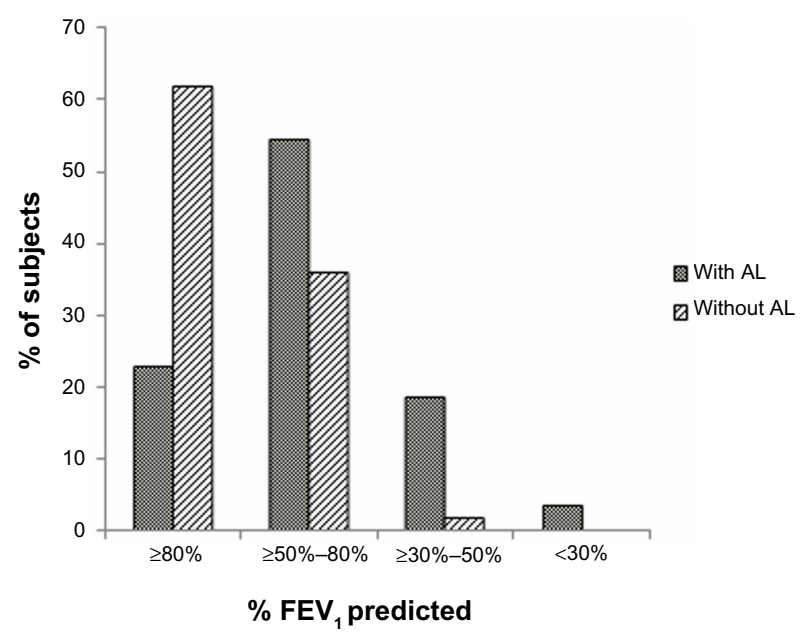

Figure I GOLD grading of patients with and without airway limitation. Abbreviations: $\mathrm{AL}$, airway limitation; $\mathrm{FEV}_{1}$, forced expiratory volume in I second; GOLD, Global initiative for chronic Obstructive Lung Disease. 
Table 3 Characteristics of patients with and without airflow limitation (prevalence of COPD)

\begin{tabular}{|c|c|c|c|}
\hline & $\begin{array}{l}\text { Airflow } \\
\text { limitation } \\
(n=269)\end{array}$ & $\begin{array}{l}\text { No airflow } \\
\text { limitation } \\
(n=726)\end{array}$ & $\begin{array}{l}\text { Airway } \\
\text { limitation } \\
\text { versus } \\
\text { no airway } \\
\text { limitation* }\end{array}$ \\
\hline Age, mean (SD) & $71.0(8.8)$ & $65.0(9.8)$ & $P<0.001$ \\
\hline Males, n (\%) & $258(96 \%)$ & $692(95 \%)$ & $P=0.689$ \\
\hline Current smoker; n (\%) & $76(28.3 \%)$ & $197(27.1 \%)$ & $P=0.726$ \\
\hline Ex-smoker; n (\%) & $193(71.7 \%)$ & $529(72.9 \%)$ & \\
\hline Pack-years; mean (SD) & $48.6(26.2)$ & $4 I . I(25.8)$ & $P<0.001$ \\
\hline BMI; mean (SD) & $23.3(3.1)$ & $25.1(3.4)$ & $P<0.001$ \\
\hline Diagnosis of COPD; $\mathrm{n}(\%)$ & $33(12.3 \%)$ & $15(2 \%)$ & $P<0.00$ I \\
\hline $\begin{array}{l}\text { Positive chronic } \\
\text { bronchitis criteria } n \text { (\%) }\end{array}$ & $68(25.3 \%)$ & 135 (I8.6\%) & $P=0.020$ \\
\hline $\mathrm{FEV}_{1}$; mean (SD) & $1.776(0.64)$ & $2.483(0.62)$ & $P<0.001$ \\
\hline $\mathrm{FEV}_{6}$; mean (SD) & $2.789(0.81)$ & $3.034(0.77)$ & $P<0.001$ \\
\hline CAT score; mean (SD) & $8.3(6.5)$ & $6.4(5.4)$ & $P<0.001$ \\
\hline \multicolumn{4}{|l|}{ CV history } \\
\hline Myocardial infarction & $4 \mathrm{I}(15.2 \%)$ & $117(16.1 \%)$ & \\
\hline Hypertension & $207(77.0 \%)$ & 529 (72.9\%) & \\
\hline Chronic heart failure & $34(12.6 \%)$ & 79 (10.9\%) & \\
\hline Angina & $46(17.1 \%)$ & $146(20.1 \%)$ & \\
\hline Arrhythmia & 59 (21.9\%) & $14 \mid(19.4 \%)$ & \\
\hline Stroke & 18 (6.7\%) & $30(4.1 \%)$ & \\
\hline Arteriosclerosis & $9(3.3 \%)$ & $15(2.1 \%)$ & \\
\hline Other CVD & $48(17.8 \%)$ & $139(19.1 \%)$ & \\
\hline Hypertension only & $110(40.9 \%)$ & $293(40.4 \%)$ & \\
\hline
\end{tabular}

Note: *Chi-squared test.

Abbreviations: CV, cardiovascular; CVD, cardiovascular disease; COPD, chronic obstructive pulmonary disease; SD, standard deviation; CAT, COPD assessment test; $\mathrm{FEV}_{1}$, forced expiratory volume in I second; $\mathrm{FEV}_{6}$, forced expiratory volume in 6 seconds.

Yamasaki et $\mathrm{al}^{12}$ in Japan, the prevalence of airflow limitation in patients with CVD was also lower $(10.9 \%$ and $22.6 \%$, respectively). These studies were both conducted on single sites. In the Wada et al study, there was good collaboration between cardiology and respirology. Additionally, although this study excluded subjects $<40$ years old, there were no

Table 4 The number of patients with airflow limitation by CV history

\begin{tabular}{lll}
\hline CV history & $\mathbf{n}$ & $\begin{array}{l}\text { Airflow limitation } \\
\mathbf{n}(\%)\end{array}$ \\
\hline Myocardial infarction & 158 & $41(25.9 \%)$ \\
Hypertension & 736 & $207(28.1 \%)$ \\
Chronic heart failure & 113 & $34(30.1 \%)$ \\
Angina & 192 & $46(24.0 \%)$ \\
Arrhythmia & 200 & $59(29.5 \%)$ \\
Stroke & 48 & $18(37.5 \%)$ \\
Arteriosclerosis & 24 & $9(37.5 \%)$ \\
Other CVD & 187 & $48(25.7 \%)$ \\
Hypertension only & 403 & $110(27.3 \%)$ \\
\hline
\end{tabular}

Abbreviations: CV, cardiovascular; CVD, cardiovascular disease. entry criteria for smoking. ${ }^{11}$ In the Yamasaki et al study there were no entry criteria for age or smoking. ${ }^{12}$

In our study, the presence of COPD was shown to increase with age, as expected, but undiagnosed airflow limitation was also seen in patients as young as $40-49$ years. There were statistically significant differences observed between patients with and without airflow limitation, for parameters including age, smoking pack-years, CAT score, chronic bronchitis, and body mass index. In patients with chronic bronchitic symptoms, $33.5 \%$ had airflow limitation, suggesting that this history may be a useful predictor of COPD amongst patients with CVD. This has also been demonstrated recently in another study, which showed that underdiagnosis of COPD was particularly a problem in younger men with chronic bronchitic symptoms - a phenotype which is not usually recognized as COPD in Japan ${ }^{25}$ because the Japanese COPD phenotype tends to be seen as: older, more emphysemic, less exacerbations, and less chronic bronchitic. Increased risk of COPD was associated with all CVDs; in our study, there was no significant difference in the number of patients with or without airflow limitation, with the CVDs of ischemic heart disease, coronary heart failure, and arrhythmias.

In this study, when patients were categorized using the GOLD criteria for airflow limitation, ${ }^{26}$ the majority (55\%) were in GOLD Grade 2, but $20 \%$ of patients had an $\mathrm{FEV}_{1}$ less than $50 \%$ of predicted, showing that even patients with severe-to-very-severe airflow limitation had not been previously diagnosed. Patients with COPD categorized as GOLD Grade 1 show an increased risk of mortality, so it is important to find and treat these undiagnosed patients. ${ }^{27}$

The prevalence of patients with airflow limitation was spread fairly evenly between the centers (split by the usage of lung function testing). As expected, there were a higher number of patients with a prior diagnosis of COPD in the centers with greater use of spirometry, compared with the centers where its use was "seldom". But even those centers classed as having using spirometry "often" had a high number of CVD patients with undiagnosed COPD.

Patients with CVD and a smoking history are at increased risk of airway limitation, and should benefit from lung function screening, as they have been reported to be frequently asymptomatic. ${ }^{28}$ The mean CAT score in patients with airflow limitation was a little higher $(8.3 \pm 6.5)$, compared with patients with no airflow limitation (6.4 \pm 5.4$)$. The score for patients with airflow limitation was lower than for patients with diagnosed COPD,${ }^{18}$ but it should be appreciated that most of the patients in our study had not been diagnosed with COPD - presumably, because their symptoms were 
inconclusive. Support for this interpretation comes from Miravitlles et al, ${ }^{29}$ who showed that St George's Respiratory Questionnaire scores in people identified as having COPD on screening were significantly lower than in those with a prior COPD diagnosis, and only a little higher than the normal range. CAT scores in CVD patients were similar to those seen in Japanese workers with undiagnosed airflow limitation, identified in a screening spirometry study. ${ }^{30}$

There are some limitations of this study. We recruited patients older than $>40$ years old with a smoking history; smoking is not prevalent among Japanese women of this age group. Further study is needed to confirm the prevalence of COPD among nonsmokers.

\section{Conclusion}

Airflow limitation compatible with COPD was seen in a quarter of outpatients with CVD in our study, with almost all of them being undiagnosed. This suggests that it is important to look routinely for COPD in CVD patients $>40$ years old with a history of smoking, as the combination of COPD with CVD is known to be associated with poorer prognosis.

\section{Acknowledgments}

The authors are grateful to the 17 medical institutes and investigators who participated in this study: Kazufumi Takeuchi (Chugoku Central Hospital); Yutaka Kajikawa (NHO Fukuyama Medical Center); Yasushi Terada (Shonai Amarume Hospital); Nobuaki Shinozaki (Shonan Atsugi Hospital); Hiroshi Shudo (Hiro Internal and Cardiology Clinic); Kenshi Fujii (Sakurabashi Watanabe Hospital); Atsuyuki Wada (Kusatsu General Hospital); Yoshihiko Araki (Osaka Prefectural Medical Center for Respiratory and Allergic Diseases); Akinori Hashimoto (Hashimoto Internal Clinic); Toshiyuki Goto (Goto Internal Clinic); Takaaki Kato (Kato Internal Clinic); Toshiaki Kadokami (Futsukaichi Hospital); Kunihiko Ueda (Ueda Internal and Cardiology Clinic); Kazuki Nakajima (Nakajima Cardiology and Internal Clinic); Shinya Okamoto (Iwasaki Hospital); Takahiro Yazu (Yazu Internal Clinic). The authors are also grateful to the Center for Clinical Trials, Japan Medical Association, and to all teams of GSK for their contribution to this study. The authors thank Diana Jones (Cambrian Clinical Associates Limited, Cardiff, UK) for medical writing and manuscript coordination, which was funded by the study sponsor.

\section{Disclosure}

The study has been funded, planned, and prepared by GlaxoSmithKline KK (GSK), who also drafted this manu- script for publishing. All authors had final responsibility for manuscript submission.

Katsuya Onishi discloses no conflict of interest. Paul W Jones discloses that his university has received honoraria and research grants from the commercial entity that sponsored the study. Gerry W Hagan discloses having received consultancy fee from GSK and reports ownership of GSK stock options. Daisuke Yoshimoto is an employee of GSK and reports ownership of GSK stock options.

\section{References}

1. Murray CJ, Lopez AD. Alternative projections of mortality and disability by cause 1990-2020: Global Burden of Disease Study. Lancet. 1997;349(9064):1498-1504.

2. Lopez AD, Shibuya K, Rao C, et al. Chronic obstructive pulmonary disease: current burden and future projections. Eur Respir J. 2006;27(2): 397-412.

3. Takahashi T, Ichinose M, Inoue H, Shirato K, Hattori T, Takishima T. Underdiagnosis and undertreatment of COPD in primary care settings. Respirology. 2003;8(4):504-508.

4. Celli BR, MacNee W; ATS/ERS Task Force. Standards for the diagnosis and treatment of patients with COPD: a summary of the ATS/ ERS position paper. Eur Respir J. 2004;23(6):932-946.

5. Omori H, Nonami Y, Mihara S, Marubayashi T, Morimoto Y, Aizawa H. Prevalence of airflow limitation on medical check-up in Japanese subjects. J UOEH. 2007;29(3):209-219.

6. Finkelstein J, Cha E, Scharf SM, et al. Chronic obstructive pulmonary disease as an independent risk factor for cardiovascular morbidity. Int J Chron Obstruct Pulmon Dis. 2009;4:337-349.

7. Sin DD, Man SFP. Chronic obstructive pulmonary disease: a novel risk factor for cardiovascular disease. Can J Physiol Pharmacol. 2005;83(1): $8-13$.

8. Mannino DM, Thorn D, Swensen A, Holguin F. Prevalence and outcomes of diabetes, hypertension, and cardiovascular disease in COPD. Eur Respir J. 2008;32(4):962-969.

9. Hawkins NM, Huang Z, Pieper KS, et al. Chronic obstructive pulmonary disease is an independent predictor of death but not atherosclerotic events in patients with myocardial infarction: analysis of the Valsartan in Acute Myocardial Infarction Trial (VALIANT). Eur J Heart Fail. 2009;11(3):292-298.

10. Soriano JB, Rigo F, Guerrero D, et al. High prevalence of undiagnosed airflow limitation in patients with cardiovascular disease. Chest. 2010; 137(2):333-340.

11. Wada $\mathrm{H}, \mathrm{Nakano} Y$, Nagao $T$, et al. Detection and prevalence of chronic obstructive pulmonary disease in a cardiovascular clinic: evaluation using a hand held $\mathrm{FEV}_{1} / \mathrm{FEV}_{6}$ meter and questionnaire. Respirology. 2010;15(8):1252-1158.

12. Yamasaki A, Hashimoto K, Hasegawa Y, et al. COPD is frequent in conditions of comorbidity in patients treated with various diseases in a university hospital. Int J Chron Obstruct Pulmon Dis. 2010;5: 351-355.

13. Agustí AG, Noguera A, Sauleda J, Sala E, Pons J, Busquets X. Systemic effects of chronic obstructive pulmonary disease. Eur Respir J. 2003; 21(2):347-360.

14. Curkendall SM, Lanes S, de Luise C, et al. Chronic obstructive pulmonary disease severity and cardiovascular outcomes. Eur J Epidemiol. 2006;21(11):803-813.

15. Donaldson GC, Hurst JR, Smith CJ, Hubbard RB, Wedzicha JA. Increased risk of myocardial infarction and stroke following exacerbation of COPD. Chest. 2010;137(5):1091-1097.

16. Dalal AA, Shah M, Lunacsek O, Hanania NA. Clinical and economic burden of patients diagnosed with COPD with comorbid cardiovascular disease. Respir Med. 2011;105(10):1516-1522. 
17. Müllerova H, Agusti A, Erqou S, Mapel DW. Cardiovascular comorbidity in COPD: systematic literature review. Chest. 2013;144(4): 1163-1178.

18. Jones PW, Harding G, Berry P, Wiklund I, Chen WH, Leidy N. Development and first validation of the COPD Assessment Test. Eur Respir J. 2009;34(3):648-654.

19. Japanese Respiratory Society. Guidelines for the Diagnosis and Treatment of COPD (3rd ed). Tokyo: Japanese Respiratory Society; 2009.

20. Vandevoorde J, Verbanck S, Schuermans D, Kartounian J, Vincken W. Obstructive and restrictive spirometric patterns: fixed cut-offs for FEV1/ FEV6 and FEV6. Eur Respir J. 2006;27(2):378-383.

21. Anthonisen NR, Connett JE, Kiley JP, et al. Effects of smoking intervention and the use of an inhaled anticholinergic bronchodilator on the rate of decline of FEV1. The Lung Health Study. JAMA. 1994;272: 1497-1505.

22. Bursi F, Vassallo R, Weston SA, Killian JM, Roger VL. Chronic obstructive pulmonary disease after myocardial infarction in the community. Am Heart J. 2010;160(1):95-101.

23. Salisbury AC, Reid KJ, Spertus JA. Impact of chronic obstructive pulmonary disease on post-myocardial infarction outcomes. Am J Cardiol. 2007;99(5):636-641.
24. Gan SC, Beaver SK, Houck PM, MacLehose RF, Lawson HW, Chan L. Treatment of acute myocardial infarction and 30-day mortality among women and men. N Engl J Med. 2000;343(1):8-15.

25. Nakano Y, Nagai A, Mishima M, Yoshimoto D, Hagan G. [Investigation of COPD management in primary care in Japan using handheld spirometer]. Kokyu. 2012;31:273-281. Japanese.

26. Global Initiative for Chronic Obstructive Lung Disease (GOLD). [cited: September 19, 2013]. Available from: http://www.goldcopd. org/. Accessed September 19, 2013.

27. Mannino DM, Doherty DE, Sonia Buist A. Global Initiative on Obstructive Lung Disease (GOLD) classification of lung disease and mortality: findings from the Atherosclerosis Risk in Communities (ARIC) study. Respir Med. 2006;100(1):115-122.

28. Ulrik CS, Løkke A, Dahl R, et al; TOP study group. Early detection of COPD in general practice. Int J Chron Obstruct Pulmon Dis. 2011;6: $123-127$.

29. Miravitlles M, Soriano JB, García-Río F, et al. Prevalence of COPD in Spain: impact of undiagnosed COPD on quality of life and daily life activities. Thorax. 2009;64(10):863-868.

30. Nishimura K, Mitsuma S, Kobayashi A, et al. COPD and disease-specific health status in a working population. Respir Res. 2012;14(1):61-66.
International Journal of COPD

\section{Publish your work in this journal}

The International Journal of COPD is an international, peer-reviewed journal of therapeutics and pharmacology focusing on concise rapid reporting of clinical studies and reviews in COPD. Special focus is given to the pathophysiological processes underlying the disease, intervention programs, patient focused education, and self management protocols.

\section{Dovepress}

This journal is indexed on PubMed Central, MedLine and CAS. The manuscript management system is completely online and includes a very quick and fair peer-review system, which is all easy to use. Visit $\mathrm{http} / / / \mathrm{www}$.dovepress.com/testimonials.php to read real quotes from published authors. 